\title{
MATHEMATICS
}

\section{Some assembly needed}

lan Stewart

\section{Origami, the ancient Japanese art of paper folding, is mathematically deeper than it looks. Delving into its complexities allows the construction of elaborate and useful structures from simple, flat templates.}

Saving space is an imperative in commerce, engineering and the structure of living creatures. Furniture and garden equipment come in flat-packs to save storage space in the warehouse and the delivery van - and, it must be said, to make the purchaser do all that timeconsuming work of assembly. The solar panels that provide satellites with electrical power have to be folded so that they fit into the bay of a space shuttle. And who could fail to be entranced by the emergence of a butterfly from a pupa, as pulsing blood inflates bedraggled, folded tissue into glorious, gaudy wings?

Over the past two decades, a dedicated band of mathematicians, engineers and computer scientists has been developing a new branch of mathematics to understand the science of folding objects flat. In a contribution ${ }^{1}$ to a recent conference $^{2}$ that exemplifies these efforts, Taketoshi Nojima details the derivation of a surprising range of geometrical forms from a flat sheet of material, including "tubes, conical shells, circular membranes, movable/shape-changeable models and highly rigid 3-D cores".

Researchers' inspiration in this area derives from two quite different sources: 'biomimetics', the technological mimicry of biological processes and structures, and the ancient Japanese art of origami. The results have been intriguing and ingenious, and have already found applications in maps, food packaging, folding drinks containers, car airbags and spacecraft antennas. Among future applications could be foldable plastic bottles, lightweight skeletons for aerospace structures and compact light-sails for interplanetary travel. The mathematics developed along the way could also feed back into a better understanding of nature's own origami: the growth and development of leaves, buds and insect wings.

Origami's potential as a source of mathematical problems has been recognized for some time. Its patterns of folds can construct geometric forms beyond those possible with Euclid's traditional rule and compass, such as regular seven-sided and nine-sided polygons. The basic problem of origami is the flat-folding problem: given a diagram of fold lines on a flat sheet of paper, can the paper be folded into a flat shape without introducing any further creases?

In 1996, two computer scientists, Barry Hayes of Placeware, Inc. and Marshall Bern at Xerox's Palo Alto Research Center, proved that this question is mathematically equivalent to a famous problem in logic, the 3-SAT problem ${ }^{3}$. This is the 'satisfiability problem', and is an example of an NP-hard problem, meaning that the running time required for any algorithm to solve the problem grows rapidly as the problem becomes more complex, so that the calculation takes too long to be feasible. The satisfiability problem asks the question whether there is some assignment of values for boolean expressions - combinations of true-orfalse logical statements - built from simple three-component formulas such that the entire expression returns the value 'true.' Mathematically, therefore, origami is deeper than it looks.

The same is true biologically, and this is where Nojima's research comes in. By studying natural folding patterns, especially those associated with the growth of plants, he has devised methods for folding remarkable threedimensional shapes from flat sheets of lightweight material. For example, one class of structures derives from the well-known occurrence of Fibonacci numbers (the sequence of numbers in which each is the sum of the 34,55 and so on) in various cylindrical or helical plants, such as pine cones and pineapples. The scales of a pine cone, for instance, form two families of helices, one twisting clockwise and the other anticlockwise. The numbers of helices in these families are two adjacent Fibonacci numbers, such as 8 and 13. Folding patterns with a similar form can be used to create rigid helical structures from flat sheets, which are rolled into a cylinder and then folded. If the sheet is first formed into a cone, collapsible conical structures can also be made (Fig. 1).

This idea is only the beginning. Far more elaborate shapes can also be constructed by origami methods. Often these resemble biological forms - flowers, horns, shells. Some of Nojima's patterns are inspired by the buds of the morning glory and the folds in an earwig's wings. Another source of inspiration is purely

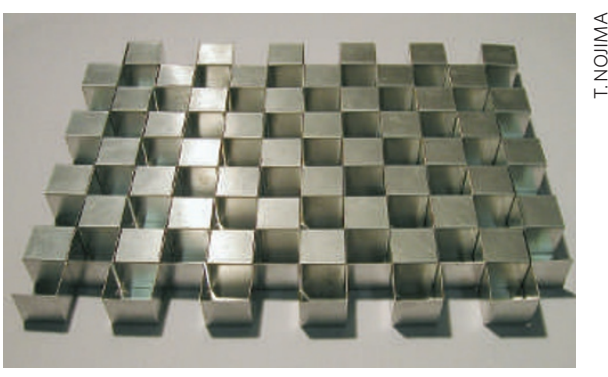

Figure 2 | Industrial application of origami. The mathematical folding schemes developed by Nojima ${ }^{1}$ can be used to stamp out tiled patterns from sheet metal, which can then be folded and welded to form rigid, lightweight materials that might be of use, for example, in the aerospace industry.

mathematical: tiling patterns, or 'tessellations', in the plane. Clever folding processes based on tessellations lead to rigid, lightweight materials of a 'honeycomb' type (Fig. 2). Some of these can be stamped out from sheet metal, folded and then welded so that they retain the folded shape, providing a simple and reliable way to manufacture apparently complicated materials. The work ${ }^{1}$ analyses the precise geometric conditions required for these constructions, putting them on a solid mathematical basis. It also pays attention to practical issues, such as the thickness of the sheet being folded.

The potential of these simple but ingenious ideas is huge. Complex three-dimensional forms could arise from flat, stamped sheets of 'shape memory' materials merely by heating them. They could also be made from flat sheets of rubber and inflated, a usefully reversible construction technique. Honeycomb cores are common in the aerospace industry for their rigidity and lightness, and any new, simple manufacturing process is likely to prove valuable. Origami structures, being lightweight and compact, are 'green': they can easily be recycled, and even if they're not, the amount of rubbish they create is reduced.

Nojima ends with a look to the future. He envisages the creation of a new discipline, which I am tempted to name 'origamics'. This would be a combination of engineering, mathematics and biology, and could lead to significant progress in a variety of fields. Nojima himself cites "plastic buckling, biomimetic robotic modelings, movable origami modeling for education or edutainment... [and] new interpretations of bionic mechanics." He could well be right.

Ian Stewart is at the Warwick Mathematics Institute, Zeeman Building, University of

Warwick, Coventry CV4 7AL, UK.

e-mail: i.n.stewart@warwick.ac.uk

1. Nojima, T. http://impact.kuaero.kyoto-u.ac.jp/pdf/ Origami.pdf

2. VIP Symp. Internet Related Res. Tokyo, 2007 http://internetconferences.net/ipsi/conference. php? conf $=52$ \&past $=1$

3. Bern, M. \& Hayes, B. Proc. 7th ACM-SIAM Symp. Discrete Algorithms 175-183 (SIAM, Philadephia, PA, 1996). 